\title{
A Signal Timing Optimization in Traffic Management using ABC Algorithm
}

\author{
R. Subashini \\ Research scholar,Mathematics \\ Theivanai Ammal College for Women \\ Villupuram 605401 T.N India
}

\author{
A. R. Rishivarman \\ Assistant Professor,Mathematics, \\ Theivanai Ammal College for Women \\ Villupuram 605401,T.N India
}

\begin{abstract}
The ABC algorithm is a new population-based meta-heuristic approach, and it is inspired by the foraging behaviour of honeybee swarm. This study discusses Artificial Bee Colony (ABC) algorithm for finding optimal setting of traffic signals in coordinated signalized networks for given fixed set of link flows. For optimizing traffic signal timings in coordinated signalized networks, ABC with GA (ABCGA) model is developed and tested. A standard traffic model is used to estimate total network performance index (PI). The ABCGA is tested in various levels with signalized road network. Results showed that the proposed model is slightly better in signal timing optimization in terms of final values of PI when it is compared with Fixed time model, and evolutionary algorithm (EA) based model. Results also showed that the ABCGA model improves network's PI when it is compared with Fixed time and EA methods.
\end{abstract}

\section{Keywords}

Artificial Bee Colony, Genetic Algorithm, signal timings, optimization.

\section{INTRODUCTION}

Artificial bee colony algorithm (ABC) developed by Karaboga and further developed by Karaboga and Basturk is a nature inspired algorithm based on the intelligent foraging behaviour of honeybee swarm. The ABC algorithm describes the foraging behaviour, learning, memorizing and information sharing characteristics of honeybees. The colony of artificial bees consists of three groups of bees: Employed bees, Onlookers bees and Scouts bees [1]. The colony of the artificial bees is divided into two groups, first half of the colony consists of the employed artificial bees and the second half includes the onlooker bees.

After the invention of $\mathrm{ABC}$ by Karaboga, the fist conference paper introducing $\mathrm{ABC}$ was published in 2006 (Basturk and Karaboga). The first journal article describing $\mathrm{ABC}$ and evaluating its performance was presented by Karaboga and Basturk, in which the performance of $\mathrm{ABC}$ was compared to GA, PSO and particle swarm inspired evolutionary algorithm. In 2008, the second article presenting a performance evaluation of $\mathrm{ABC}$ was published by Karaboga and Basturk [1]. In 2009, a public domain web-site

(http://mf.erciyes.edu.tr/abc) dedicated to ABC was constructed. There are several source codes, written in different programming languages, of $\mathrm{ABC}$ and many publications about the modifications to $\mathrm{ABC}$ and their applications are presented in the website. The main algorithm of $\mathrm{ABC}$ is relatively simple and its implementation is, therefore, straightforward for solving optimization problems and $A B C$ has been found to be very effective in the studies above, being able to produce very good results at a low computational cost. Therefore, after these initial publications many studies have been carried out on $\mathrm{ABC}$.

\section{WORKING PRINCIPLE OF ABC}

The foraging behaviors of honeybees have recently been one of the most interesting research areas in swarm intelligence. Some approaches have been proposed to model the specific intelligent behaviours of honeybee swarms and they have been applied for solving optimization problems. Lucic and Teodorovic in [2] and Teodorovic in [3] suggested to use bee swarm intelligence aimed at solving complex problems in traffic and transportation. Teodorovic and Dell'Orco in [4] proposed the bee colony optimization to solve combinatorial problems characterized by uncertainty, as well as deterministic combinatorial problems. Teodorovic and Dell'Orco in [5] presented an application of the bee colony optimization, efficient in solving the ride-matching problem. Their results showed that proposed metaheuristic appears very promising, and indicated that the development of new models based on swarm intelligence principles could significantly contribute to the solution of a wide range of complex engineering and management problems. The Artificial Bee Colony (ABC) algorithm is a new population-based metaheuristic approach proposed by Karaboga in [6] and further developed by Karaboga and Basturk [7, 8, 9]. It is inspired by the foraging behavior of honeybee swarm. The foraging bees are classified into three categories employed, onlookers and scouts. Half of the bee colony consists of employed bees, and another half consists of onlookers. In $\mathrm{ABC}$ algorithm, the position of a food source represents a possible solution to the optimization problem and the nectar amount of a food source corresponds to the quality (fitness) of the associated solution [10].The number of employed bees or the onlooker bees is equal to the number of solutions in the population. Employed bees are responsible for searching available food sources and gathering required information. They also pass their food information to onlooker bees. The onlookers select good food sources from those found by the employed bees to further search the foods. When the quality of the food source is not improved through a predetermined number of cycles, the food source is abandoned by its employed bee. In this case, the employed bee becomes a scout and starts to search for a new food source in the vicinity of the hive. In $\mathrm{ABC}$ algorithm, each cycle of the search consists of three steps. At the initialization step, the $\mathrm{ABC}$ algorithm generates a randomly distributed initial population as number of $S N$, where $S N$ denotes the number of employed bees or onlooker bees. Each initial solution $x_{i}(i=1,2, \ldots, S N)$ is a $D$ dimensional vector which $D$ is the number of decision variables of a given optimization problem. At the first step of the cycle, employed bees come into the hive and share with the bees waiting on the dance area information about nectar sources. A bee waiting on the dance area is called onlooker, 
and is responsible for making decision about the choice of a food source. At the second step, an onlooker chooses a food source area depending on the nectar information distributed by the employed bees on the dance area. As the nectar amount of a food source increases, the probability of choice of that food source increases as well. The determination of a new food source is carried out by the bees based on a visual comparison process of positions of food sources. At the third step of the cycle, when a food source is abandoned by the bees, a new food source is randomly determined by a scout bee and replaces the abandoned one. These three steps are repeated until a predetermined number of cycles, called Maximum Cycle Number $(M C N)$, is reached. An onlooker bee chooses a food source depending on the probability value, $p_{i}$, as follows:

$p_{i}=\frac{\text { fit }_{i}}{\sum_{n=1}^{S N} \text { fit }_{n}}$

Where $f i t_{i}$ is the fitness value of solution $i$. In this way, the employed bees exchange their information with the onlookers. In order to share the information of nectar amount of the food sources, the employed bees use a proportional selection method known as "roulette wheel selection".

In order to produce a candidate food location from the old one in population, the following equation is used.

$v_{i j}=x_{i j}+\varphi_{i j}\left(x_{i j}-x_{k j}\right)$

where $v_{i j}$ is the candidate food position which can replace the old one in the memory and $\emptyset_{i j}$ is a random number generated in the interval $[-1,1]$. The values $k=1,2, \ldots, S N$ and $j=1,2, \ldots, D$ are randomly chosen indexes. Of course, $k$ must be different from $i$, to avoid that old and new location coincide, in order to find food sources having more nectar amount than the old one. The parameter $\emptyset_{i j}$ controls the production of neighbour food sources around $x_{i j}$, and represents the visual comparison of two food positions carried out by a bee. If a parameter value determined using Eq. (2) exceeds the constraints of the decision variables, the parameter is set to its upper and lower boundary, depending on which constraint has been exceeded.

As mentioned above, the food source abandoned by the bees is replaced with a new food source by the scouts at the third step of the cycle. In ABC algorithm, this is simulated by generating a random location and replacing the abandoned one with it. If a location cannot be further improved in a predetermined number of cycles, then that food source is assumed to be abandoned. The value of predetermined number of cycles, called "limit", is an important control parameter of the algorithm. Karaboga and Akay proposed to determine this value as $S N * D$ [10]. This operation can be done using Eq. (3).

$x_{i}^{j}=x_{\text {min }}^{j}+\operatorname{rand}[0,1]\left(x_{\text {max }}^{j}-x_{\text {min }}^{j}\right)$

After each candidate source location $v_{i j}$ is generated, its performance is compared with that of the old one. If the new food source has equal or better nectar than the old source, it replaces the old one in the memory. Otherwise, the old one is retained in the memory. In other words, a greedy selection mechanism is employed as selection between the old and the candidate location.

The steps of the $\mathrm{ABC}$ algorithm are given as follows:

Initialize all parameters;
Repeat while Termination criteria is not meet

Step 1: Employed bee phase for computing new food sources.

Step 2: Onlooker bees phase for updating location the food sources based on their amount of nectar.

Step 3: Scout bee phase for searching new food sources in place of rejected food sources.

Step 4: Memorize the best food source identified so far.

End of while

Output: The best solution identified so far.

\section{MODEL FORMULATION:}

The proposed ABC GA model consists of two main parts namely $\mathrm{ABC}$ algorithm and $\mathrm{GA}$ traffic model. $\mathrm{ABC}$ algorithm optimizes traffic signal timings under fixed set of link flows. GA traffic model is used to compute PI, which is called objective function, for a given signal timing and staging plan in network. The network Disutility Index (DI), one of the Transport model, PI, is used as objective function. The DI is a measure of disadvantageous operation; that is stops, delay, fuel consumption, etc. The standard DI is linear combination of delay and stops. The objective function and corresponding constraints are given as: $\quad P I=\underset{\psi, \mathrm{q}=\text { fixed }^{\text {Min }}}{D I=}$ $\sum_{a \in L}\left[W_{d_{a}} \cdot d_{a}(\psi)+K \cdot W_{s_{a}} \cdot S_{a}(\psi)\right]$

Subject to

$$
\begin{gathered}
\\
\psi(c, \theta, \varphi) \in \Omega_{0}= \\
\left\{\begin{array}{cc}
c_{\min } \leq c \leq c_{\max } & \text { cycle time constraints } \\
0 \leq \theta \leq c & \text { value off set constraints } \\
\varphi_{\min } \leq \varphi \leq c & \text { green time constraints } \\
\sum_{i=1}^{z}(\varphi+I)_{i}=c & -
\end{array}\right\}
\end{gathered}
$$

where $d a$ is delay on link $a$ ( $L$ set of links), $w_{d a}$ is linkspecific weighting factor for delay $d, K$ is stop penalty factor to express the importance of stops relative to delay, $S a$ is stop on link $a, w_{s a}$ is link-specific weighting factor for stops $S$ on link $a, q$ is fixed set of link flows, $\psi$ is signal setting parameters, $c$ is common cycle time (sec), $\theta$ is offset time (sec), $\varphi$ is green time (sec), $\Omega_{0}$ is feasible region for signal timings, $I$ is intergreen time (sec), and $z$ is number of stages at each signalized intersection in a given road network.

The green timings can be distributed to all signal stages in a road network according to the following in order to provide the cycle time constraint.

$$
\begin{gathered}
\varphi_{i}=\varphi_{\min , i}+\frac{p_{i}}{\sum_{k=1}^{z} p_{i}}\left(c-\sum_{k=1}^{Z} I_{k}-\sum_{k=1}^{Z} \varphi_{\min , k}\right) \\
i=1,2, \ldots z
\end{gathered}
$$

where $\varphi_{i}$ is the green time (sec) for stage $i, \varphi_{\min , i}$ is minimum green time (sec) for stage $i, p i$ is generated randomly green timings (sec) for stage $i, z$ is the number of stages and $I$ is intergreen time $(\mathrm{sec})$ between signal stages and $c$ is the common cycle time of the network (sec).

\section{ABC WITH GENETIC ALGORITHM (ABC GA)}

The mutation and crossover in artificial bee colony are used to achieve the optimization in ABC GA algorithm. It can avoid local minima for complex optimization problems. However, the optimization process of traditional ABC algorithm is slow. The genetic algorithm is use to revise the nectar position in 
the process of foraging to improve the convergence precision based on $\mathrm{ABC}$.

\subsection{Encoding of bee}

The encoding of bee colony is real-number. Each dimension of employ bees presents the green time of each phase. The length is the number of phase. It can be expressed by formula (7).

$\left[g_{1}, g_{2}, \ldots g_{i}, \ldots g_{I}\right]$

\subsection{Steps of the algorithm}

\subsubsection{Initial algorithm parameters}

Those parameters are needed to be initialized, such as the position of nectar, population size $s$, number of onlookers $N_{c}$ , number of scout $N_{r e}$, migration times $N_{e d}$ and the probability of migration $P_{e d}$.

\subsubsection{Foraging process}

i. The employee bees are tumbled and moved with unit vector.

ii. The employee bees are scout until the fitness can't be improved.

The employ bees are tumbled and scout to update the position by the formula (8)

$\theta^{i}(j+1, k, l)=\theta^{i}(j, k, l)+c(i) \emptyset(i)$

$\emptyset(i)=\frac{\Delta(i)}{\sqrt{\Delta^{T}(i) \Delta(i)}}$

$\theta^{i}(j, k, l)$ presents the employ bees $i$ position of the $j^{\text {th }}$ nectar, the $k^{t h}$ reproduction and the $l$ th migration. $c(i)$ is the cycle step. $\emptyset(i)$ is the direction vector of unit. $\Delta(i)$ is the random vector.

iii. Mutation

$\theta_{\text {gbest }}$ is defined as the global optimum. $\theta_{\text {lbest }}$ is the current position. $P$ is the variation operator and is expressed by formula (10).

$P=\theta_{r 1}+F\left(\theta_{\text {gbest }}-\theta_{r 2}\right)+\operatorname{rand}\left(\theta_{\text {lbest }}-\theta_{r 3}\right)$

where, $F$ is the zoom factor, $F \in[0.5,1] . \theta_{r 1}, \theta_{r 2}, \theta_{r 3}$ are the random individual bees in the employees population.

iv. Crossover

The crossover is executed by formula (11) to increase the employee bees population.

$\theta_{i}=\left\{\begin{array}{lr}P_{i} \text { if rand }<C R \text { or } i=\text { irand } \\ X_{i} & \text { otherwise }\end{array}\right.$

where, $C R$ is the cross factor. Irand is the random latitude. Rand is the random number, rand $\in[0,1]$.

\subsubsection{Reproductive behaviour}

The $S / 2$ employbees with worst fitness become scout and the $S / 2$ bees with the best fitness can proceed.

\subsubsection{Migration behaviour}

The employee bees with worst fitness are eliminated with probability $P_{e d}$ and they become scout.

In the ABC GA, optimization steps can be given in the following way:

Step 0: Initialization. Define the user specified parameters; the number of decision variables (n) (this number is sum of the number of green times as stage numbers at each intersection, the number of offset times as intersection numbers and common cycle time), the constraints for each decision variable, the size of bee colony $(S N)$, maximum cycle number $(M C N)$.

Step 1: Set $t=1$.

Step 2: Generate the random initial signal timings within the constraints of decision variables.

Step 3: Distribute to the green timings to the stages according to distribution rule as mentioned above. At this step, randomly generated green timings at Step 2 are distributed to the stages according to generated cycle time at the same step, minimum green and intergreen time.

Step 4: Get the network data and fixed set of link flows for ABC GA traffic model.

Step 5: Run ABC GA.

Step 6: Get the network PI. At this step, the PI is determined using ABC GA traffic model.

Step 7: If $t=M C N$ then terminate the algorithm; otherwise, $t=t+1$ and go to Step 2 .

\section{SIMULATION ANALYSIS}

In order to prove the validity of the model ABC GA, some intersection of Villupuram is selected to simulation. This intersection has four phases. The simulative is done in MATLAB7.0. In simulation process the number of employee bee is equal to 50 .The times of tumble $N_{C}$ is equal to 100 . The time of scout produce $N_{r e}$ is equal to 5.The time of elimination $N_{e d}$ is equal to 3 . The elimination probability $P_{e}$ is equal to 0.3 . The green time of straight phase is between 6 and 90. The green time of left phase is between 6 and 40.The cycle is between 36 and 200. The signal timing optimization model is simulated many times by ABC GA algorithm under low demand, medium demand and high demand. Meanwhile, the simulation is done by the fixed time method. The total delay vehicles in 10 cycles of 10 simulation times is presented. It is pointed that the application effects of the model and the ABC GA proposed is much better than the other under high demand. It is found that the average delay vehicles is reduced by $25 \%$ than the fix time method and reduced by $5 \%$ than the Evolutionary algorithm. ABC GA converges faster and can get the better solution than the Evolutionary algorithm for the signal timing optimization. 
Table 1. Signal Timing when Comparing Performance

\begin{tabular}{|c|c|c|c|c|c|c|c|c|}
\hline \multirow{2}{*}{$\begin{array}{c}\text { Demand } \\
\text { scenarios }\end{array}$} & $\begin{array}{c}\text { The } \\
\text { Optimization } \\
\text { algorithm }\end{array}$ & $\begin{array}{c}\text { Phase } \\
1(\mathrm{~s})\end{array}$ & $\begin{array}{c}\text { Phase } \\
2(\mathrm{~s})\end{array}$ & $\begin{array}{c}\text { Phase } \\
3(\mathrm{~s})\end{array}$ & $\begin{array}{c}\text { Phase } \\
4(\mathrm{~s})\end{array}$ & $\begin{array}{c}\text { Cycle } \\
\text { time }(\mathrm{s})\end{array}$ & $\begin{array}{c}\text { Average } \\
\text { delay } \\
\text { vehicles } \\
\text { (vech) }\end{array}$ & $\begin{array}{c}\text { Generation } \\
\text { appearing the } \\
\text { best fitness }\end{array}$ \\
\hline $\begin{array}{c}\text { Low- } \\
\text { Demand }\end{array}$ & FIX TIME & 30 & 26 & 35 & 25 & 116 & 16.1 & - \\
\cline { 2 - 9 } & EA & 50 & 12 & 28 & 15 & 105 & 14.2 & 55 \\
\cline { 2 - 9 } & ABCGA & 41 & 10 & 32 & 12 & 95 & 13.1 & 40 \\
\hline \multirow{2}{*}{$\begin{array}{c}\text { Medium- } \\
\text { Demand }\end{array}$} & FIX TIME & 38 & 32 & 45 & 35 & 150 & 46.2 & - \\
\cline { 2 - 9 } & EA & 45 & 13 & 59 & 6 & 123 & 40.4 & 60 \\
\hline \multirow{2}{*}{$\begin{array}{c}\text { High- } \\
\text { Demand }\end{array}$} & FIX TIME & 60 & 30 & 53 & 32 & 175 & 122.2 & - \\
\cline { 2 - 9 } & EA & 62 & 13 & 57 & 24 & 156 & 95.1 & 65 \\
\cline { 2 - 9 }
\end{tabular}

\section{APPLICATIONS OF ABC}

Since its origination, the $\mathrm{ABC}$ algorithm has become extraordinarily fashionable as it has less number of control parameters; it is robust and easy to apply. It is successfully applied to the problems from different application areas by many researchers. Applications of $\mathrm{ABC}$ algorithms listed by [11] with subject area of use. The $\mathrm{ABC}$ algorithm has application in field of computer science, electrical engineering, mechanical engineering and electronics engineering. Artificial bee colony algorithm applied for feature selection [12], multicast routing [13], single machine scheduling [14], balancing in assembly line [15], designing of controller [16] and to find solution of travelling salesman problem [17]. List of applications of $\mathrm{ABC}$ algorithm are endless, there are almost every field including engineering, science and management where $\mathrm{ABC}$ algorithm is in use with priority over other competitive optimization algorithm.

\section{CONCLUSION}

The urban intersection is often very crowd in cities, in this paper it is proposed a signal timing optimization model ABC GA. Because the throughput of the intersection is the most important under congested conditions, the delay vehicles of a cycle time are chosen as the performance indexes of model. ABC with GA is proposed to solve the Problem. Computational experiments of an intersection in Villupuram City are conducted to validate the model formulations and solution algorithms. The result of Simulation shows the validity of this model and method. It reduces the total delay vehicles and especially works well in high demand. It can improve traffic capacity of intersections. In our future work, we are planning to improve further level of optimization in a crowded cities using various optimization algorithms.

\section{ACKNOWLEDGMENT}

The authors are thankful to referees for their valuable comments and suggestions for improving the present paper.

\section{REFERENCES}

[1] Dervis Karaboga, Beyza Gorkemli, Celal Ozturk, Nurhan Karaboga. "A comprehensive survey: artificial bee colony (ABC) algorithm and applications". 2012.

[2] Lucic, P. and Teodorovic, D. (2002). "Transportation modelling : an artificial life approach". in ICTAI, 216223.

[3] Teodorovic, D. (2003). "Transport modeling by multiagent systems: a swarm intelligence approach". Transportation Planning and Technology, 26(4), 289312.

[4] Teodorovic, D. and Dell'Orco, M. (2005). "Bee colony optimization-a cooperative learning approach to complex transportation problems", 10th EWGT Meeting, in: Poznan, September 3-16, 2005.

[5] Teodorovic, D. and Dell'Orco, M. (2008). "Mitigating Traffic Congestion: Solving the Ride-Matching Problem by Bee Colony Optimization". Transportation Planning and Technology, 31(2), 135-152.

[6] Karaboga, D. (2005). "An idea based on honeybee swarm for numerical optimization”. Technical Report TR06, Erciyes University, Engineering Faculty, Computer Engineering Department, Turkey.

[7] Karaboga, D. and Basturk, B. (2007a). "A powerful and efficient algorithm for numerical function optimization: artificial bee colony (ABC) algorithm". Journal of Global Optimization, 39, 459-471.

[8] Karaboga, D. and Basturk, B. (2007b). "Artificial bee colony (ABC) optimization algorithm for solving constrained optimization problems". Lecture Notes in Artificial Intelligence 4529, pp. 789-798, SpringerVerlag, Berlin.

[9] Karaboga, D. and Basturk, B. (2008). "On the performance of artificial bee colony (ABC) algorithm". Applied Soft Computing, 8, 687-697. 
[10] Karaboga, D. and Akay, B. (2009). "A comparative study of artificial bee colony algorithm". Applied Mathematics and Computation, 214, 108-132.

[11] Mansouri, P., B. Asady, and N. Gupta. "The BisectionArtificial Bee Colony algorithm to solve Fixed point problems." Applied Soft Computing 26 (2015): 143-148.

[12] Yavuz, Gürcan, and Doğan Aydin. "Angle modulated Artificial Bee Colony algorithms for feature selection." Applied Computational Intelligence and Soft Computing 2016 (2016): 7

[13] Marinakis, Yannis, Magdalene Marinaki, and Athanasios Migdalas. "A Hybrid Discrete Artificial Bee Colony Algorithm for the Multicast Routing Problem." Applications of Evolutionary Computation. Springer International Publishing, 2016. 203-218.

[14] Yurtkuran, Alkın, and Erdal Emel. "A discrete artificial bee colony algorithm for single machine scheduling problems." International Journal of Production Research (2016): 1-19.

[15] Saif .U "Hybrid Pareto artificial bee colony algorithm for assembly line balancing with task time variations." International Journal of Computer Integrated Manufacturing (2016): 1-16.

[16] Caraveo .C, Fevrier Valdez, and Oscar Castillo. "Optimization of fuzzy controller design using a new bee colony algorithm with fuzzy dynamic parameter adaptation." Applied Soft Computing 43 (2016): 131142.

[17] Shailesh Pandey and Sandeep Kumar, "Enhanced Artificial Bee Colony Algorithm and It's Application to Travelling Salesman Problem," HCTL Open International Journal of Technology Innovations and Research, Vol 2, March 2013, Pages 137-146, ISSN: 2321-1814, ISBN: 978-1-62776-111-6. 